\title{
EMBEDDED ZEROTREE WAVELET CODING WITH JOINT HUFFMAN AND ARITHMETIC CODING
}

\author{
Harmandeep Singh Nijjar ${ }^{1}$, Charanjit Singh ${ }^{2}$ \\ ${ }^{I}$ MTech, Department of ECE, Punjabi University Patiala \\ ${ }^{2}$ Assistant Professor, Department of ECE, Punjabi University Patiala
}

\begin{abstract}
Image compression is a task of reducing the image size without image quality degradation. It provides solution to both the problems of limited storage and long transmission time. This paper presents a method of combinational coding using Huffman and Arithmetic technique. The Embedded Zerotree Wavelet method which provides embedded bit stream is employed for image compression. The EZW symbols obtained are first coded using Huffman method. The Huffman bit stream is converted into byte stream and then into equivalent decimal. The final integer stream obtained is coded using Arithmetic coding. The proposed method of joint coding provides improved compression ratio as compared with Huffman coding alone.
\end{abstract}

Keywords: Wavelet Transform, EZW, Huffman coding, Arithmetic coding, PSNR $* * *$

\section{INTRODUCTION}

Interest in digital image processing is rapidly increasing as it's applications are extensively reaching out into our day today lives. The major fields with applications of digital image processing are entertainment, medicine, security, industry etc. The sub fields of image processing are image segmentation, image de-noising, image compression, pattern detection and image enhancement [1]. As everybody is adopting the digital techniques, the main problem associated with such transition to digital methods is limited storage space and long transmission time. Huge amount of storage is required to store digital images used in various fields and time required to transmit such images is also very long. There is a single solution to both these problems, i.e. Image Compression. Image compression field deals with reducing the size of digital images while maintaining the acceptable image quality. The compression is achieved by removing the redundancies present in the images. The different redundancies present in the images are:

1. Inter-pixel redundancy: Redundancy due to similar values of neighbouring pixels in an image.

2. Psycho-visual Redundancy: Redundancy due to pixel values which are not identifiable for human eyes.

3. Coding Redundancy: Redundancy due to longer code word length for a pixel.[2]

The types of image compression are broadly divided into following two categories: a) Lossless Compression: In this type of image compression, whole of the information present in the image is retained and compression is obtained only by reducing the code word length [3]. The commonly used coding methods are Huffman coding, Run Length coding, Arithmetic coding, LZW coding etc.

b) Lossy Compression: In lossy compression, information loss occurs to achieve the compression. The information loss is due to the quantization step which is irreversible [4].The information loss is compensated by high compression ratios achieved by this method.

\section{WAVELET TRANSFORM}

Wavelet transform is used in image compression field to convert an image from spatial domain to frequency domain as it becomes easy to manipulate and change image properties in frequency domain. Wavelet transform helps in identifying and removing the inter-pixel redundancies present in an image. In wavelet transform, the signal is represented using the wavelets. The additional advantage of wavelet transform as compared to other transforms is that it provides both time and frequency information along with multi-resolution analysis [5]. The basic working of wavelet transform for images is that images are passed through a high pass and low pass filter simultaneously. This divides the image into high and low frequency components resulting in approximate and detail coefficients as shown below: 

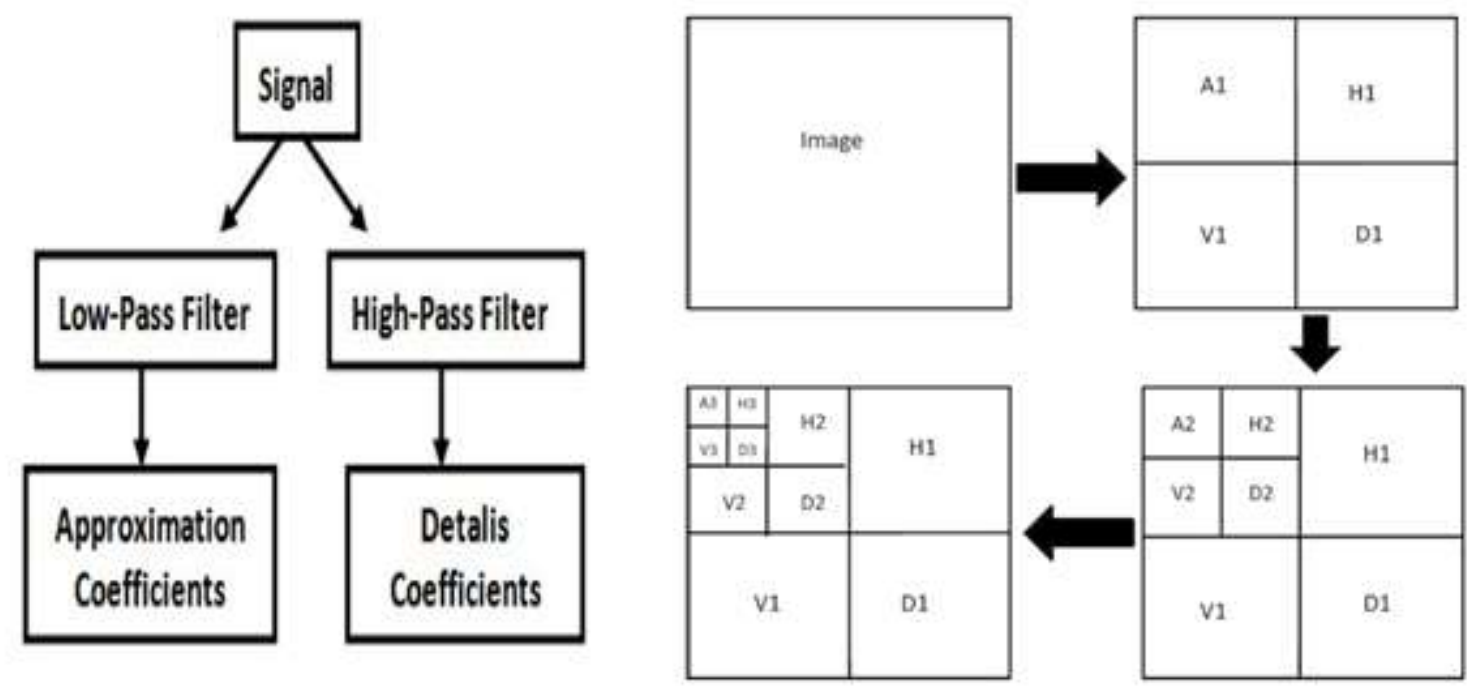

Fig. 1(a): Wavelet Decomposition into Levels Fig. 1(b): Wavelet Decomposition at Different Different Frequency Components

The A1 are approximate components which are low frequency components and H, D1 and V1 are horizontal, diagonal and vertical detail components [6]. Approximate components are most important as human eyes are most sensitive to low frequency components.

\subsection{Embedded Zerotree Wavelet}

Embedded Zerotree Wavelet coding method introduced by J. Shapiro in 1993 provides an embedded bit stream for wavelet coefficients in which the most important coefficients are coded first followed by less important coefficients [7]. This coding method provides progressive coding with accuracy increasing as more bits are added to bit stream. Even if the bandwidth is limited, still good image can be obtained with progressive coding technique as compared to other techniques.

The EZW method works by selecting a threshold. The threshold is selected as $\mathrm{T}=2^{\log _{2} \operatorname{Max}(\mathrm{f}(\mathrm{x}, \mathrm{y})}$ where $\operatorname{Max}(\mathrm{f}(\mathrm{x}$, $y)$ is maximum image coefficient value. The coefficients of image are scanned in mortan fashion and each coefficient is compared with threshold and placed into one of the four groups:

1) Positive Significant $(P)$ : If coefficient value is positive and greater than the threshold.

2) Negative Significant (N): If coefficient value is negative and greater than the threshold.

3) Isolated Zero (Z): If coefficient value is less than threshold but have significant child coefficients at other resolutions.

4) Zerotree Root (T): If coefficient value is less than threshold and have no significant child coefficients at other resolutions.[8]

Once a zerotree root has been identified, the child coefficients of that root are not required to be coded. Also, mostly coefficients found during scanning are zerotree roots and it's child. The method involves two passes, i.e. Dominant Pass and Subordinate Pass. Dominant pass distribute the coefficients into one of the four groups and Subordinate pass increases the accuracy of $\mathrm{P}$ and $\mathrm{N}$ coefficients [7]. After these two passes, the threshold value is halved and process is repeated. This procedure continues until the final threshold value is reached.

Huffman Coding: Huffman coding is a probability based coding which provides variable length code words to symbols based on the probability of their occurrence. The symbols having lower occurrence probability are coded with longer code words and symbols with higher occurrence probability are coded with shorter code words. [9]

Arithmetic Coding: Arithmetic coding is similar to Huffman coding with assigning shorter code for symbols with high occurrence probability and longer codes to the symbols with low occurrence probability. The difference from Huffman coding is that instead of assigning code to each symbol in a message [10], it encodes the whole message with single codeword.

\section{PROPOSED METHOD}

The proposed image compression method uses the EZW image compression method. The coefficients obtained using the EZW method are coded using Huffman coding scheme. The Huffman coded bit stream is further coded using Arithmetic coding. 


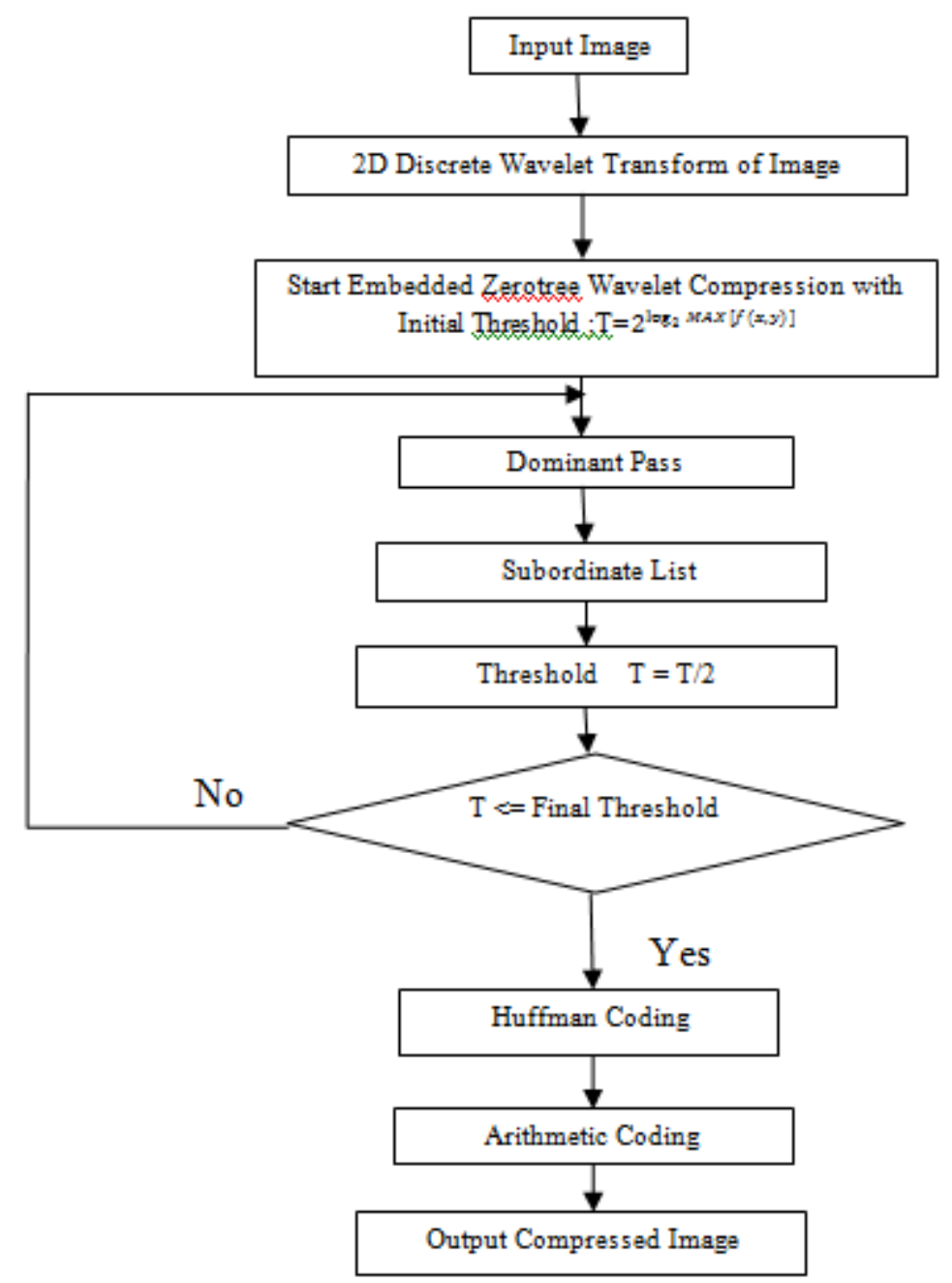

Fig.2: Flowchart of the Proposed Method

The working of dominant pass is explained below:

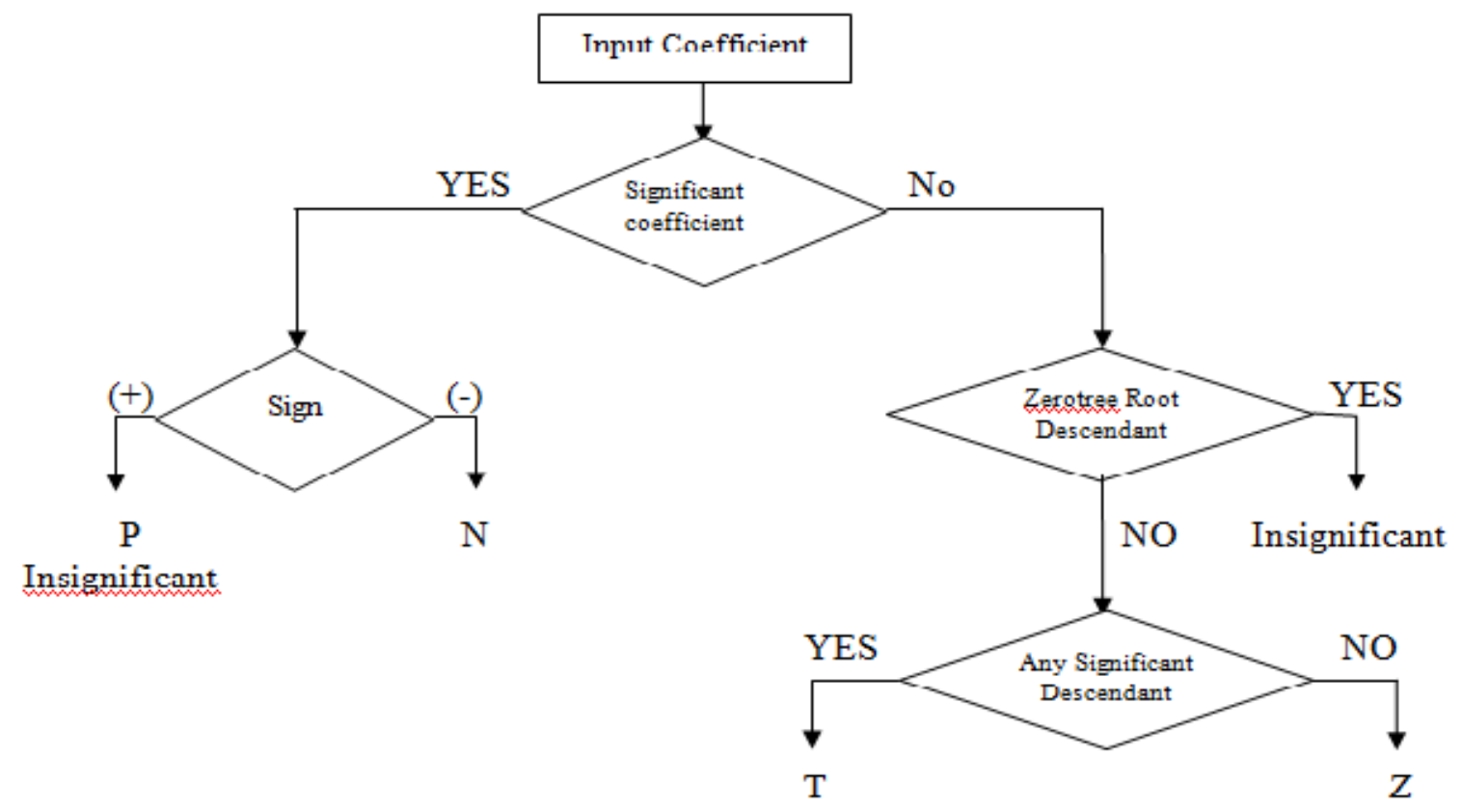

Fig.3: Flowchart for Dominant Pass 
Steps:

1. Input image is converted to gray-scale image.

2. 2D Discrete Wavelet Transform is performed on grayscale image.

3. The Embedded Zerotree Wavelet coding method is performed to achieve compression.

a. Final threshold is selected.

b. Dominant and subordinate pass is performed for each threshold until the final threshold value is reached.

4. The EZW bit stream is coded using Huffman coding method. The Huffman coding method uses the maximum presence of zerotree and isolated zeros present and assign the mentioned codes to the different symbols. ' $T$ ' is coded as ' 0 ', ' $\mathrm{Z}$ ' is coded as ' 10 ', ' $\mathrm{N}$ ' is coded as ' 110 ', ' $\mathrm{P}$ ' is coded as ' 1110 ' and ' 11111 ' to indicate the end of bit stream

5. The Huffman coded bit stream is converted to byte stream by grouping the 8 consecutive bits and then converting the bytes into their decimal equivalent. This results in a sequence of integers.

6. The byte stream consisting of integers in then coded using Arithmetic coding.

\subsection{Performance Parameters}

The following parameters are used to measure the performance of the proposed algorithm:

1. Compression Ratio: It is the ratio of original image size to the compressed image size [1].

2. Peak Signal to Noise Ratio: It is the given as

PSNR $=20 \log _{10} \frac{255}{\sqrt{M S E}} \quad$ where $\quad$ MSE $=$
$\frac{1}{\mathrm{MN}} \sum_{\mathrm{y}=1}^{\mathrm{M}} \sum_{\mathrm{x}=1}^{\mathrm{N}}\left[\mathrm{I}(\mathrm{x}, \mathrm{y})-\mathrm{I}^{\prime}(\mathrm{x}, \mathrm{y})\right]^{2}$

MSE is Mean Square Error and is used to find error in compressed image [1].

\section{RESULTS}

The proposed method is applied on two different black and white images at different final threshold values. The results obtained are shown below:

\section{Image1: 'Cameraman'}
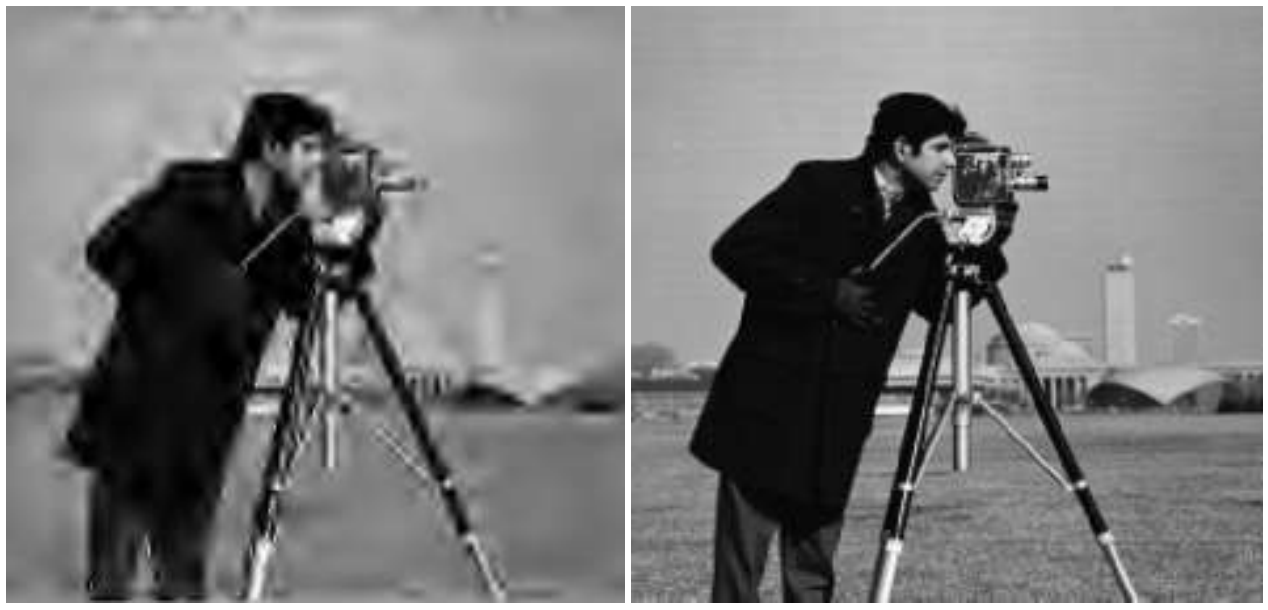

Fig.4 (a) Original Image 'Cameraman'

Fig. 4(b) 'Cameraman’ EZW Compression at Threshold = 1
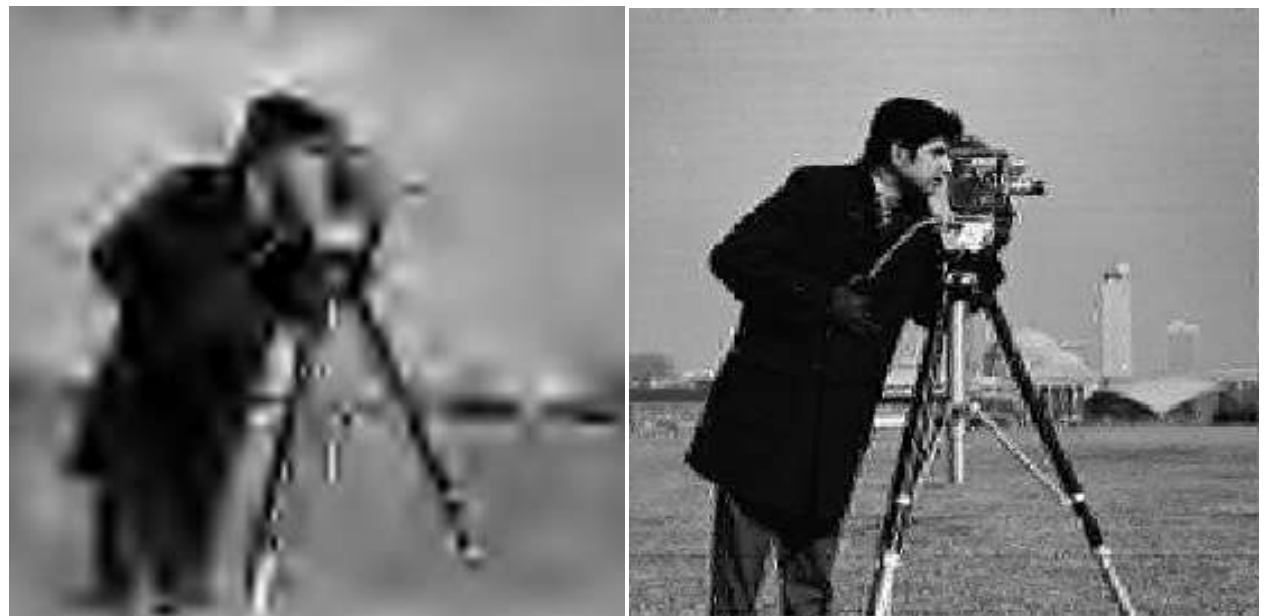

Fig. 4(c) ‘Cameraman’ EZW Compression at Threshold = 0.4 Fig. 4 (d) ‘Cameraman’ EZW Compression at Threshold = 0.1 


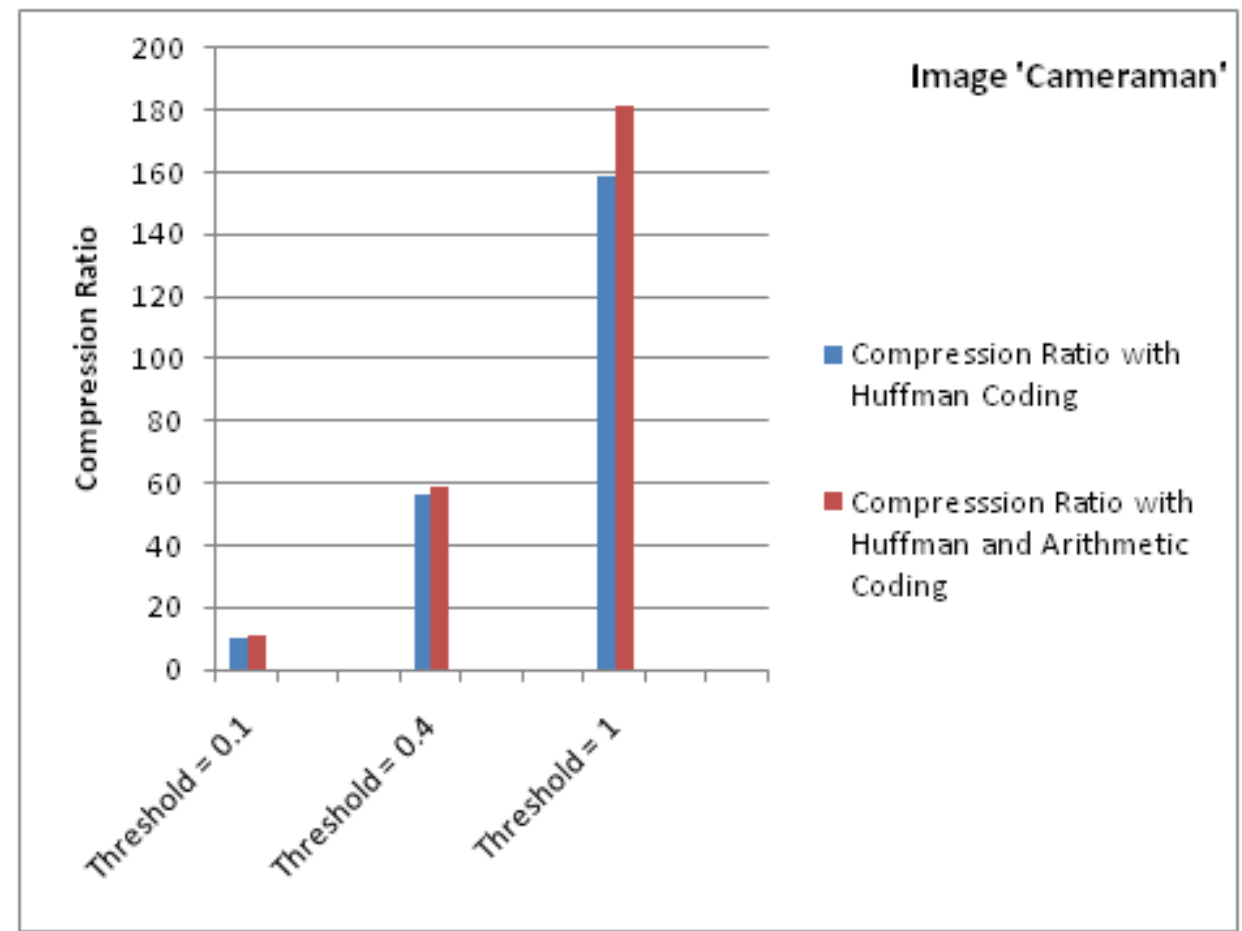

Fig. 5: Compression Ratio Comparison of EZW method with Huffman Coding and joint Huffman and Arithmetic Coding for Image 'Cameraman'.

Table 1: Performance Comparison of EZW compressing method with Huffman coding and Combinational Huffman-Arithmetic

\begin{tabular}{|c|c|c|c|c|}
\hline $\begin{array}{l}\text { Final } \\
\text { EZW } \\
\text { Threshold }\end{array}$ & $\begin{array}{l}\text { Mean Square } \\
\text { Error }\end{array}$ & $\begin{array}{l}\text { Peak Signal to } \\
\text { Noise Ratio } \\
\text { (in dB) }\end{array}$ & \begin{tabular}{lr}
\multicolumn{2}{l}{ Compression Ratio } \\
(EZW & Compression \\
with & Huffman \\
Coding) &
\end{tabular} & $\begin{array}{l}\text { Compression Ratio } \\
\text { (EZW Compression with Joint } \\
\text { Huffman and Arithmetic Coding) }\end{array}$ \\
\hline 1 & 4760.20 & 11.35 & 159.1 & 181.5 \\
\hline 0.4 & 1394.38 & 16.69 & 56 & 58.6 \\
\hline 0.1 & 775.09 & 19.24 & 10.1 & 10.9 \\
\hline
\end{tabular}

\section{Image 2: 'Man'}
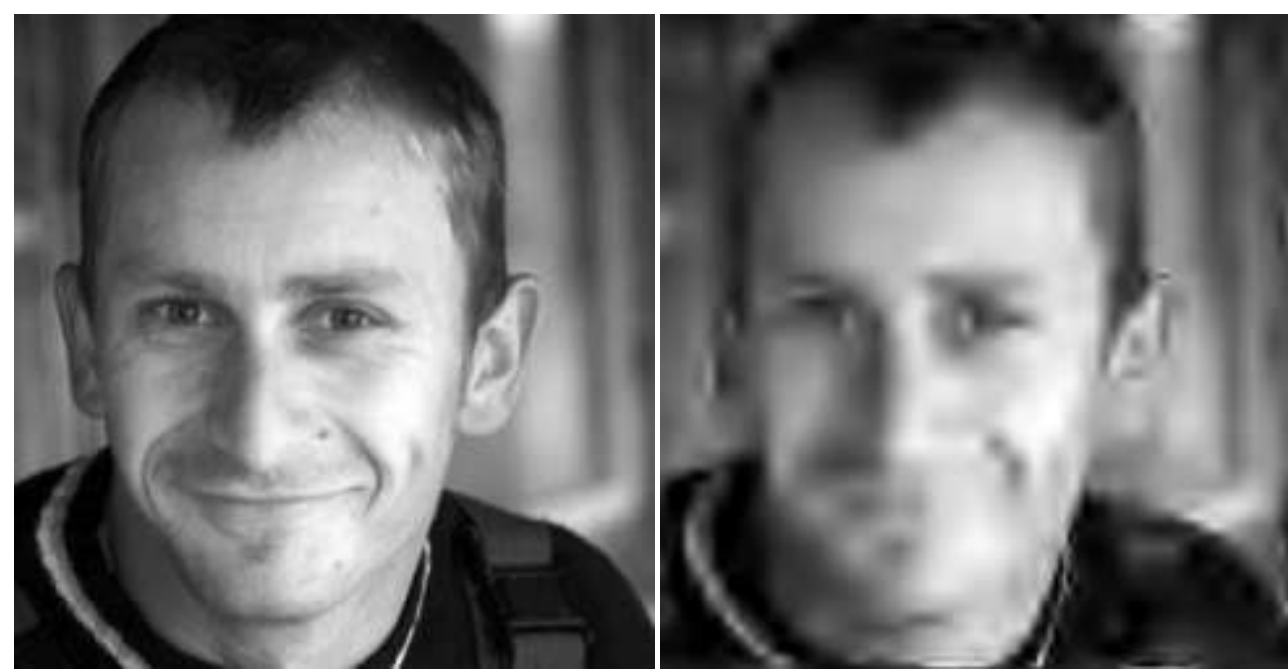

Fig.6 (a) Original Image 'Man'

Fig. 6 (b) 'Man’ EZW Compression at Threshold = 1 

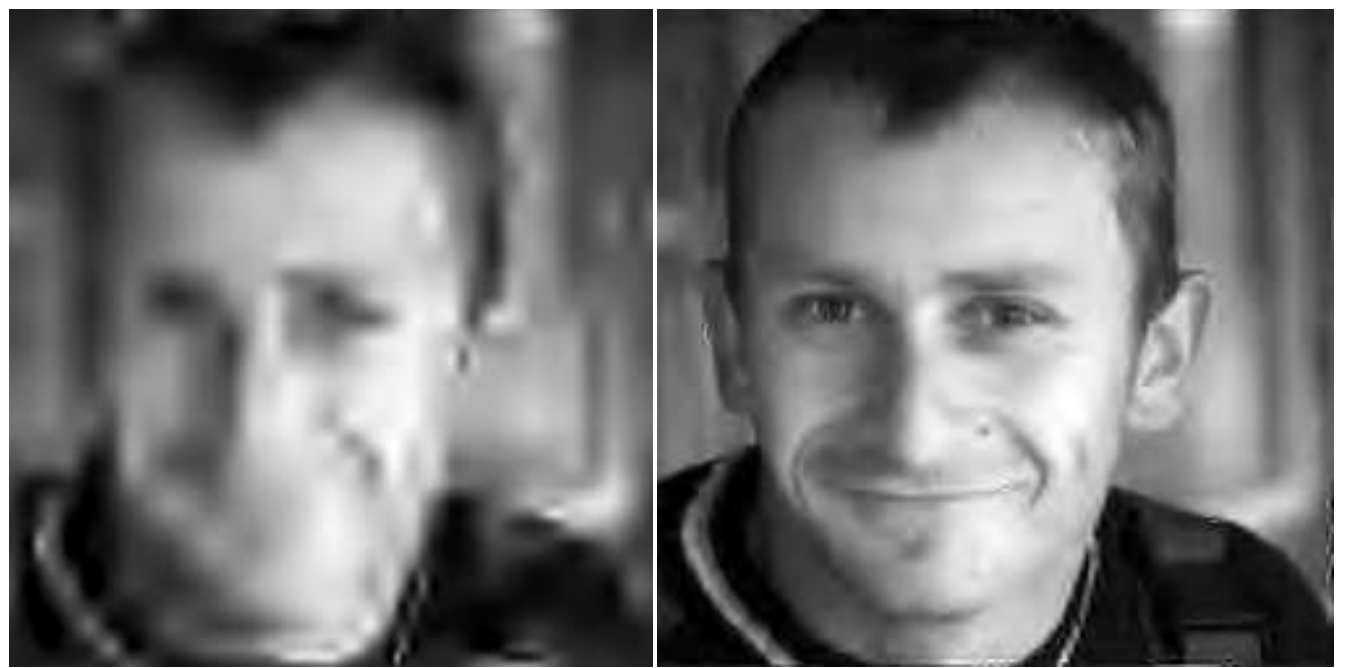

Fig. 6 (c) ‘Man’ EZW Compression at Threshold $=0.4 \quad$ Fig. 6 (d) 'Man’ EZW Compression at Threshold $=0.1$

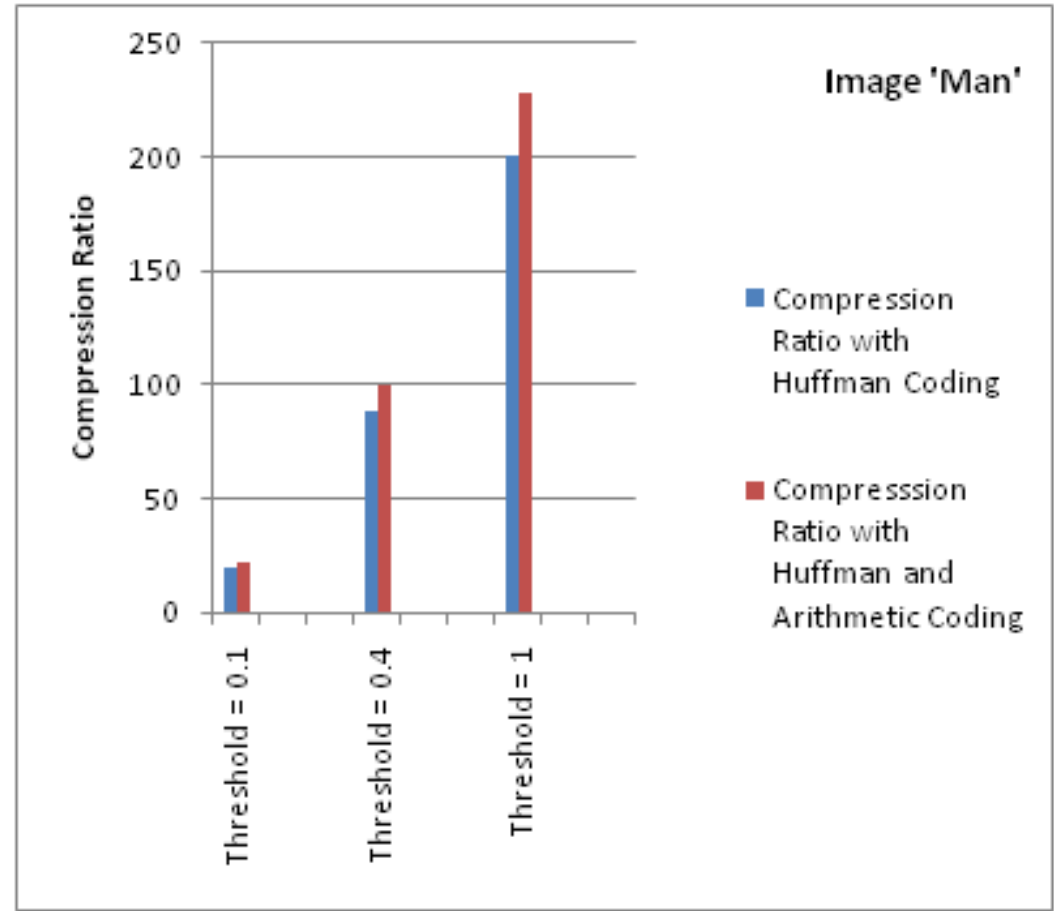

Fig. 7: Compression Ratio Comparison of EZW method with Huffman Coding and Joint Huffman and Arithmetic Coding for Image 'Man'.

Table 2: Performance Comparison of EZW compressing method with Huffman coding and Combinational Huffman-Arithmetic coding.

\begin{tabular}{|l|l|l|l|l|}
\hline $\begin{array}{l}\text { Final EZW } \\
\text { Threshold }\end{array}$ & $\begin{array}{l}\text { Mean Square } \\
\text { Error }\end{array}$ & $\begin{array}{l}\text { Peak Signal to } \\
\text { Noise Ratio (in } \\
\text { dB) }\end{array}$ & $\begin{array}{l}\text { Compression Ratio } \\
\text { (EZW Compression } \\
\text { with Huffman Coding) }\end{array}$ & $\begin{array}{l}\text { Compression Ratio } \\
\text { (EZW Compression } \\
\text { with Joint Huffman and } \\
\text { Arithmetic Coding) }\end{array}$ \\
\hline 1 & 2203.52 & 14.70 & 200.4 & 228.3 \\
\hline 0.4 & 1008.96 & 18.09 & 88.3 & 99.9 \\
\hline 0.1 & 168.88 & 25.85 & 19.5 & 22.1 \\
\hline
\end{tabular}




\section{CONCLUSION}

The proposed method when applied on two different images reveals that the joint Huffman-Arithmetic coding technique provides increased compression ratio as compared to Huffman coding alone. The compression ratio difference of Huffman coding and joint Huffman-Arithmetic coding achieved is larger for the higher threshold values and this difference decreases as the final EZW threshold is decreased. The common limitation of Arithmetic coding is time taken to encode the symbols. However, this limitation does not affect the performance of this method as initially all the symbols are coded using Huffman method which requires less time and then the arithmetic coding is used. This also helps in reducing coding time limitation of arithmetic coding as only few symbols are needed to be coded using Arithmetic method.

\section{REFERENCES}

[1]. R. C. Gonzalez, R. E. Woods, "Digital Image Processing", 2nd Ed., Prentice Hall, 2002.

[2]. V. Bastani, M. S. Helfroush and K. Kasiri, "Image Compression Based On Spatial Redundancy Removal And Image Inpainting”, Journal of Zhejiang University, Volume 11, Issue 2 , pp 92-100, February 2010.

[3]. M. Yang and N. Bourbakis, "An Overview of Lossless Digital Image Compression Techniques", 48th Midwest Symposium on Circuits and Systems, pp. 1099 -1102, USA 2005.

[4]. M. Hemalatha and S. Nithya, "A Thorough Survey on Lossy Image Compression Techniques" , International Journal of Applied Engineering Research. Vol. 11, No. 5, pp. 3326-3329, 2016.

[5]. M. A. Zahhad, S. M. Ahmed, and A. Zakaria, "An Efficient Technique for Compressing ECG Signals Using QRS Detection,Estimation, and 2D DWT Coefficients Thresholding" Modelling and Simulation in Engineering, 2010.

[6]. L. Bo and Y. Zhaorong, "Image Compression Based on Wavelet Transform", 2012 International Conference on Measurement, Information and Control, pp. 145-148, China, May 2012.

[7]. J. M. Shapiro, "Embedded Image Coding Using Zerotrees of Wavelet Coefficients", IEEE Transactions On Signal Processing, Vol. 41, No. I2, December 1991.

[8]. R. George and Manimekalai, "A Novel Approach for Image Compression Using Zero Tree Coding”, International Conference on Electronics and Communication System (ICECS ), pp. 1-5, Coimbatore, February 2014.

[9]. J. H. Pujar and L. M. Kadlaskar, "A New Lossless Method of Image Compression and Decompression Using Huffman Coding Technique", Journal of Theoretical and Applied Information Technology, Vol. 15, No. 1, 2010.

[10]. R. C. Chen, P. Y. Pai, Y. K. Chan and C. C. Chang, "Lossless Image Compression Based on Multiple-Tables Arithmetic Coding," Mathematical Problems in Engineering, 2009.

[11]. R. Janaki and A. Tamilarasi, "Still Image Compression by Combining EZW Encoding with Huffman Encoder", International Journal of Computer Applications, pp. 1-7, Vol. 13, No. 7, January, 2013. 